\title{
The approximate location of imperfection in a unit circle using the spectrum of Laplace operator as a research tool
}

\section{Introduction}

In faults diagnosis, there are a lot of methods for localizing and identify the defects $[1,2]$. Among these methods, we can distinguish non-destructive detection of defects (Non Destructive Testing, NDT) involving the comparison of the responses of the system with known force with similar characteristics of the reference model - these methods do not require destroying the tested system. NDT is carried out either at random and locally, mainly after the discovery of damage, or periodically in order to minimize the risk of failure of the object. NDE techniques are used mainly in order to characterize the type and size of likely damage locations. At the design stage not only the physical properties that we want to measure are examined, but also the geometry of the object. It has an impact on the deployment of the sensors with which we record the measurements [3].

\section{Theoretical issue}

In theoretical considerations concerning the problem of the location of the imperfection for the fixed bounded domain we will take advantage of spectral theory results and, more precisely, the conclusion of the spectral theorem for compact and self-adjoint operators, which says that all eigenvalues of the Laplace operator on the bounded $+\infty$ domain are positive, have finite multiplicities and $\Omega \subseteq \mathbb{R}^{2}$ is the limit point of eigenvalues. Consider the problem

$$
A u=\lambda u
$$

* AGH University of Science and Technology, Department of Automatics and Biomedical Engineering, Krakow, Poland 
There exists the inverse operator for the $A$ operator, therefore we have:

$$
u=A^{-1} \lambda u
$$

From the linearity of the $A^{-1}$ operator results the following:

$$
u=\lambda A^{-1} u
$$

hence:

$$
A^{-1} u=\frac{1}{\lambda} u
$$

For the above equation exists the countable set of eigenvalues $\left\{\frac{1}{\lambda_{1}}, \frac{1}{\lambda_{2}}, \frac{1}{\lambda_{3}}, \ldots\right\}$, $\lambda_{i} \neq 0, i \in \mathbb{N}$ whose the only limit point is point 0 . Furthermore, every eigenvalue has a finite multiplicity.

\subsection{The definition of the problem for the domain without the imperfection}

Let the domain $\Omega \subset \mathbb{R}^{2}$ be given. It could be a square, a circle or any other two-dimensional figure. For the $\Omega$ domain and for the $k=1,2, \ldots$ we define the problem:

$$
\begin{cases}A u_{k}^{0}(x, y)=\lambda_{k}^{0} u_{k}^{0}(x, y) & \text { in } \Omega \\ u_{k}^{0}(x, y)=0 & \text { on } \partial \Omega\end{cases}
$$

where $A$ is a linear operator $A: H_{0}^{1}(\Omega) \rightarrow H^{-1}(\Omega)$ such that the operator $-A$ is an elliptic operator and an operator inverse $A^{-1}: L^{2}(\Omega) \rightarrow L^{2}(\Omega)$ is a compact operator. Eigenvalues and eigenfunctions $\left(\lambda_{k}^{0}, u_{k}^{0}(x, y)\right)$ of the problem (5) refer to the domain without deformation.

\subsection{The definition of the problem for the domain with the imperfection}

Let us consider the $\Omega$ domain with the $D$ imperfection. The imperfection is a circle with radius $r=0.01$. Let us mark the $\Omega$ domain with described imperfection as $\Omega_{D}$. Then we get $\Omega_{D}=\Omega / D$ and we consider the following spectral problem for the $\Omega_{D}$ domain and $k=1,2, \ldots$

$$
\begin{cases}A u_{k}(x, y)=\lambda_{k} u_{k}(x, y) & \text { in } \Omega_{D} \\ u_{k}(x, y)=0 & \text { on } \partial \Omega_{D}\end{cases}
$$


The solution to the problem above is an infinite sequence of pairs $\left(\lambda_{k}, u_{k}\right)$ for $k=1,2, \ldots$, where $\lambda_{k}$ are eigenvalues and $u_{k}(x, y)$ are eigenfunctions. These eigenvalues are dependent on the location and size of the imperfection $D$.

\subsection{The definition of the inverse problem}

The main aim of our study is to solve the inverse task [4, 5], which consists in localizing the imperfection (coordinates $\left.\left(x_{0}, y_{0}\right)\right)$ in the unit circle $S^{1}=((0,0), 1)$ based on the spectrum of the Laplace operator for this circle:

$$
\sigma(A) \rightarrow(x, y)
$$

\section{Numerical simulation}

The computer simulation was carried out in MATLAB program using the PDE Tool package [5]. In the mathematical model the Laplace equation defined for the $\Omega=S^{1}((0,1), 1)$ domain was taken as an elliptic operator [7]:

$$
A u(x, y):=\frac{\partial^{2}}{\partial x^{2}} u(x, y)+\frac{\partial^{2}}{\partial y^{2}} u(x, y)
$$

The goal of the numerical simulation is to observe the behaviour of the spectrum of the Laplace operator for the unit circle when the deformation is located at different points of this circle. Because studying the spectrum of the Laplace operator for the unit circle with deformation at every point of this circle is a complicated task, we have chosen a grid of equidistant points (Fig. 1) to create the isochors maps for minimum and maximum values of this spectrum (Fig. 2c and Fig. 3c).

More precisely, we were analysing the spectrum for every subsequent point, which we placed the deformation in (Fig. 1). For every point (deformation), we used the undermentioned algorithm to get the maximum and minimum values of eigenvalues to it.

The algorithm:

1. A unit circle is used as domain $\Omega$.

2. The deformation is placed in the fixed point of this circle.

3. The deformation is placed in the fixed point of this circle (triangulation of the domain with deformation).

4. The type of the equation and its specification is chosen (eigenmodes, $a=1.0, c=0.0$, $d=1.0)$.

5. Eigenvalues $\lambda_{\min }$ and $\lambda_{\max }$ are obtained. 


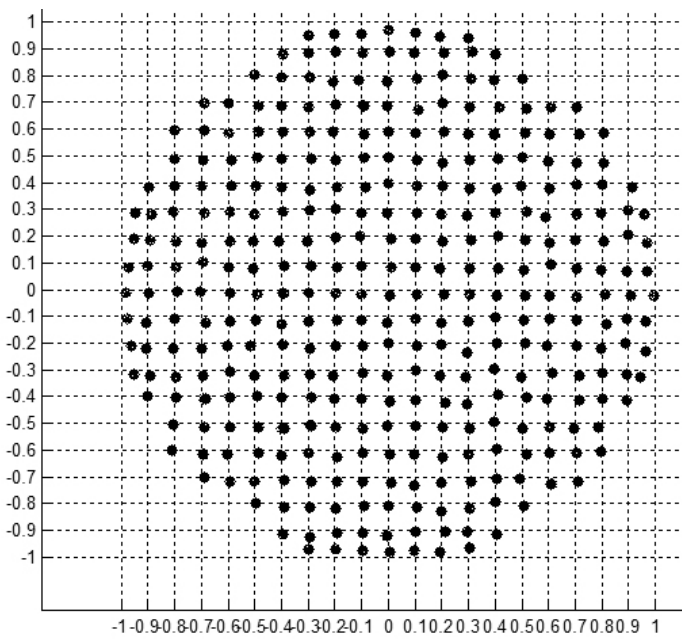

Fig. 1. The grid of points which the deformation was placed in

a)

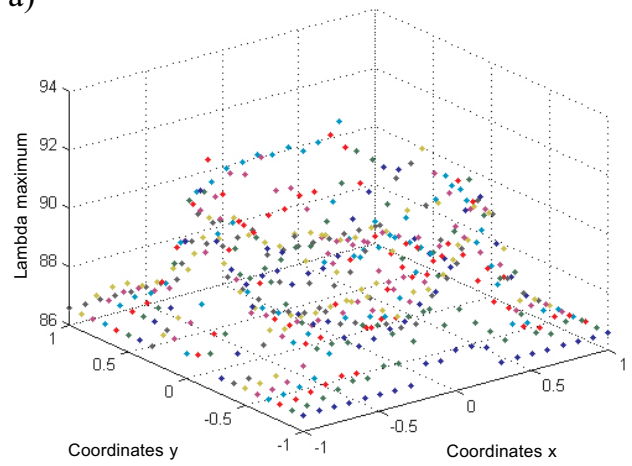

b)

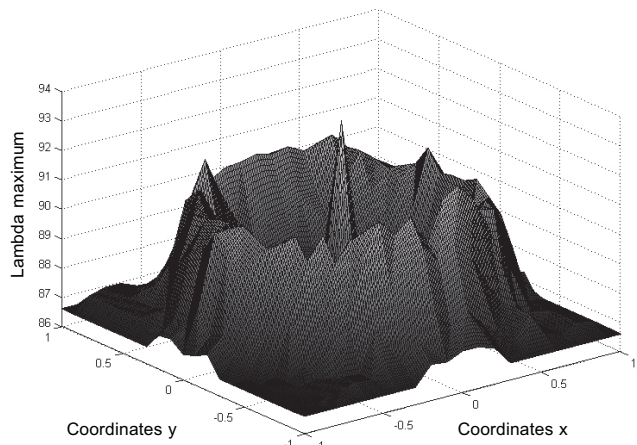

c)

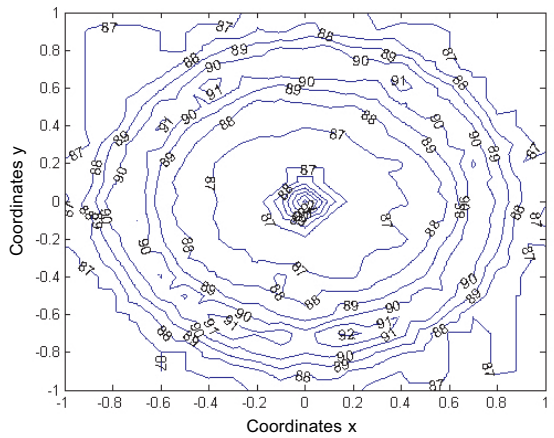

Fig. 2. Next steps in creating isochors map for $\lambda_{\max }$ : a) scattered data of $\lambda_{\max }$;

b) three-dimensional surface of $\lambda_{\max }$; c) isochors of lambda max $I_{\max }$ 
Figure 2a presents scattered data which we get for minimum values of eigenvalues $\lambda_{\text {min }}$. From this data we make a three-dimensional surface (Fig. 2b). Now that the data is in a gridded format, we compute and plot the contours of isochors (lines connecting points of the same value Figure 2c). We will use this map to read the approximate location of the imperfection.

The same analysis will be carried out for the minimum values $\lambda_{\min }$ of the spectrum of the Laplace operator. Figure 3a presents scattered data for minimum values of eigenvalues $\lambda_{\min }$. From this data we make a three-dimensional surface (Fig. 3b). Figure 3c presents the projection of the three-dimensional surface of minimum values of eigenvalues $\lambda_{\text {min }}$ on XY plane.

Now, when we have isochors of maximum and minimum values of eigenvalues, we are able to determine the domains, where the deformation will be located in. These domains will be common parts (rings) of isochors maps $I_{\min }$ and $I_{\max }$ which correspond to the measurement $\lambda_{\min }$ and $\lambda_{\max }$.

a)

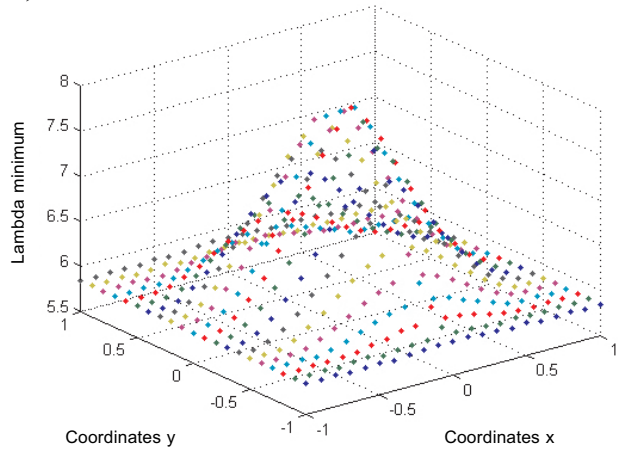

b)

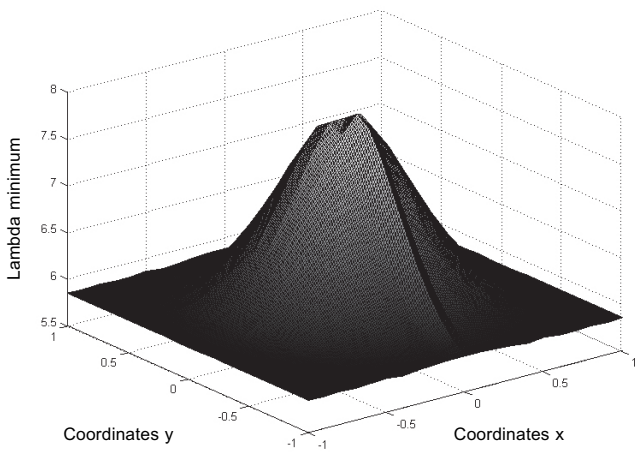

c)

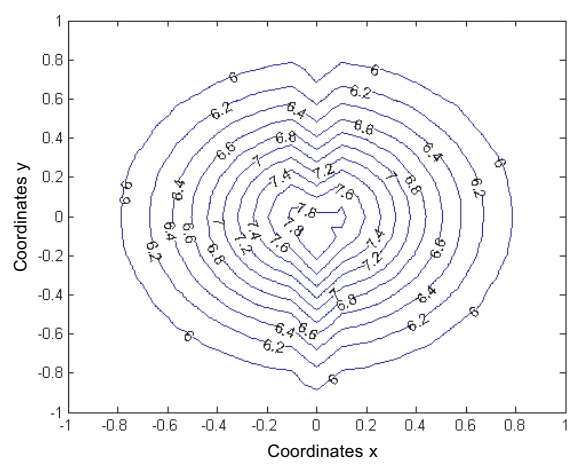

Fig. 3. Next steps in creating the isochors map for lambda min: a) scattered data of $\lambda_{\min }$;

b) three-dimensional surface of $\lambda_{\min }$; c) isochors of lambda min $I_{\min }$ 


\subsection{Example}

As an illustrative example (Fig. 4), let us consider a spectrum of form $[6.77,52.53$, $53.84,87.43]$. For this measurement, the maximum value of eigenvalues is $\lambda_{\max }=87.43$, and the minimum value of eigenvalues is $\lambda_{\min }=6.77$. From the isochors map $I_{\max }$ for $\lambda_{\max }$ we get the domain (ring) with the range [87.88]. For minimum value of eigenvalues from the isochors map $I_{\min }$ we get the domain (ring) with the range [6.6, 6.8]. Common parts of both domains (grey color in Fig. 4) are an approximate domain where imperfection is.

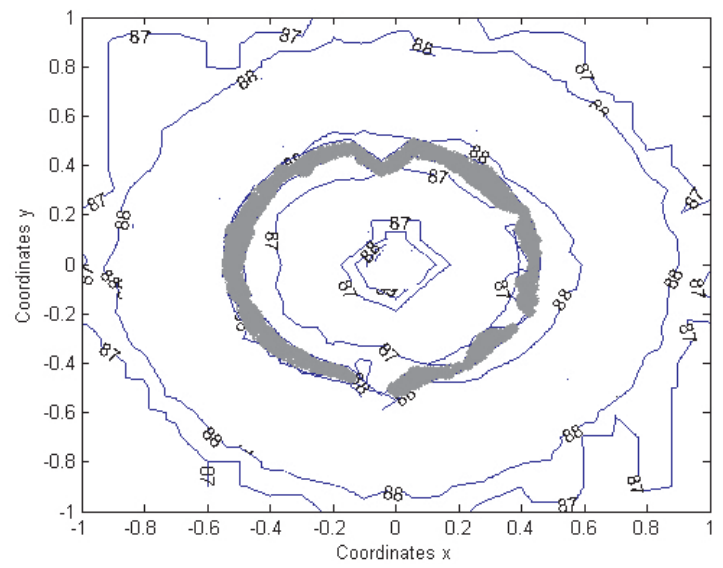

Fig. 4. An illustrative example for the spectrum of form $[6.77,52.53,53.84,87.43]$

\section{Conclusions}

As the numerical simulation shows, based on the spectrum of the Laplace operator for the unit circle $S^{1}=((0,0), 1)$ with the deformation, we are able to define domains (rings), where the deformations are located in. This method does not solve the problem, unfortunately, a clear inverse searching damage, but greatly simplifies the search for further damage.

\section{References}

[1] Uhl T., Współczesne metody monitorowania i diagnozowania konstrukcji, Wydział Inżynierii Mechanicznej i Robotyki, Akademia Górniczo-Hutnicza, www.fundacjarozwojunauki.pl/res/Tom2/ 6 Uhl.pdf.

[2] Brzęk M., Mitkowski W., Lokalizacja uszkodzeń w zadanym obszarze z wykorzystaniem teorii spektralnej, Pomiary, Automatyka, Kontrola 2014, Vol. 60, No. 1.

[3] Lipnicka M., Approximate localisation of imperfections in fixed domain, Journals of the Polish Mathematical Society 2011, Vol. 39, No. 2. 
[4] Jackowska-Strumiłło L., Sokołowski J., Żochowski A., Henrot A., On Numerical Solution of Shape Invers Problem, Computational Optimization and Application 2002, Vol. 23, pp. 231-255.

[5] Kabanikhin S.I., Definitions and examples of invers and ill-posed problems, Journal of Inverse and Ill-Posed Problems 2008, Vol. 16, No. 4, pp. 317-357.

[6] Matlab, Partial differential equation toolbox. User's guide, The Math Works, Inc. www.mathworks.com.

[7] Gilbarg D., Trudinger N.S., Elliptic Partial Differential Equations of Second Order, Springer-Verlag, Berlin - Heidelberg - New York - Tokyo, 1983. 